\title{
CORRIGENDUM
}

\section{The evolution of large-scale motions in turbulent pipe flow - CORRIGENDUM}

\author{
Leo H. O. Hellström, Bharathram Ganapathisubramani \\ and Alexander J. Smits
}

doi:10.1017/jfm.2015.418, Published by Cambridge University Press, 19 August 2015

Recent investigations by Hellström have uncovered certain errors in the papers by Hellström \& Smits (2014) and Hellström, Ganapathisubramani \& Smits (2015), which are corrected below.

Hellström \& Smits (2014) estimated the convective displacement between two consecutive data snapshots, based on the bulk velocity, to be $0.48 R$ and $0.96 R$ for Reynolds numbers 47000 and 93000 , respectively. These displacements should be corrected to $2.20 R$ and $4.40 R$. The convective length scales were not used as a part of the analysis and so the errors have no further impact on this work and do not affect its conclusions.

The incorrect estimation of the convective displacement propagated to Hellström et al. (2015), where the displacement should be corrected from $0.96 R$ to $4.77 R$ for $R e_{D}=104000$. This correction implies that the large-scale structures remain spatiotemporally correlated for longer than the reported 1-2R. Figure 1 shows the corrected temporal autocorrelation of the first proper orthogonal decomposition (POD) mode and third azimuthal mode number, $\Phi_{n}(m ; r)=\Phi_{1}(3 ; r)$, for Reynolds numbers 52000 and 104000 . The decay to the $\mathrm{e}^{-1}$ point suggests that the streamwise spatio-temporal length of the coherent structures is approximately $3 R$, which is in good agreement with the streamwise extent of the corresponding conditional mode, $\Psi_{(3,1)}$.

The corrections to the estimated spatio-temporal length scale do not further impact the work, including the conclusion that these structures of $O(3 R)$ in length are a basic building block that line up to create longer structures similar to the very large-scale motions (VLSMs).

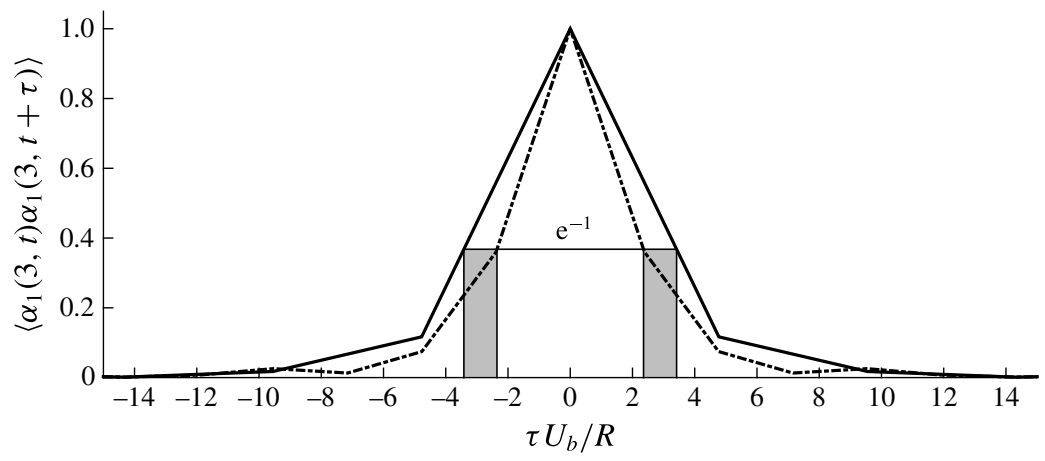

FIGURE 1. Autocorrelation of $\alpha_{(n=1)}(m=3, t)$, revealing the temporal extent of $\Phi_{1}(3 ; r)$ before a modal transition occurs: $-\cdot-\cdot-, R e_{D}=52000 ;-, R e_{D}=104000$. Shaded area shows the convective length for which the correlations fall below $\mathrm{e}^{-1}$, indicating the likely length of the meandering coherent structure. 


\section{REFERENCES}

Hellström, L. H. O., Ganapathisubramani, B. \& Smits, A. J. 2015 The evolution of largescale motions in turbulent pipe flow. J. Fluid Mech. 779, 701-715.

HellströM, L. H. O. \& SMits, A. J. 2014 The energetic motions in turbulent pipe flow. Phys. Fluids 26 (12), 125102. 